\title{
Letter to the Editor: Living Donor Liver Transplantation During the COVID-19 Pandemic: An Evolving Challenge
}

\author{
Haris Muhammad ${ }^{1} \cdot$ Duha Zaffar $^{2} \cdot$ Aniqa Tehreem $^{3}$
}

Received: 21 August 2021 / Accepted: 28 August 2021 / Published online: 10 September 2021

(c) The Society for Surgery of the Alimentary Tract 2021

\section{To the Editor:}

We enjoyed reading the article by Bhatti et al. in the recent issue of Journal of Gastrointestinal Surgery. ${ }^{1}$ The authors have described their experience and outcome of living donor liver transplant (LDLT) during the COVID-19 pandemic.

We thank the authors for addressing this important topic. However, few questions need to be addressed before reaching a conclusion. The study population was younger in both the groups pre-COVID ( $45.5 \pm 14.1$ years $)$ vs COVID (47.6 \pm 11.6 years, $P=0.3$ ) which makes them less prone to have comorbidities and therefore detrimental effects from COVID. Therefore, it is a potential bias when considering the severity of the disease and outcomes. Furthermore, warm ischemia time and duration of surgery were significantly longer in the COVID group. However, despite this, greater graft dysfunction was reported in the pre-COVID sample. This is contrary to the published data where longer warm ischemia time is associated with ischemia-reperfusion injury (IRI) which results in graft dysfunction after liver transplantation. ${ }^{2}$ Thus, it needs further clarification.

Another important clarification needed is the dosing of immunosuppressants and steroid. It is worth knowing if the steroid dose was increased in the post-COVID group to help/prevent further infection and whether mycophenolate (if used was discontinued) as it has been reported an independent predictor of severe COVID-19 in liver transplant (LT) patients. ${ }^{3}$ Interestingly, the majority of patients were males in both groups pre-COVID (130 (80.2\%)) vs COVID (45 (84.9\%), and the median MELD score was low

Aniqa Tehreem

aniqatehreem@gmail.com

1 Department of Medicine, Greater Baltimore Medical Center, Towson, MD, USA

2 Government Medical College, Srinagar, Jammu and Kashmir, India

3 Department of Medicine, Mercy Hospital (Catholic Health), Buffalo, NY, USA in both groups pre-COVID (18 (12.7-24)) vs COVID (16 (10.5-19.5)). Therefore, the results of the study cannot be generalized on the general population undergoing LDLT.

About $41 \%$ candidates with MELD $>20$ underwent LT in pre-COVID compared to $24.5 \%$ in COVID group. Subsequently, in the subgroup analysis, 30-day patient survival was better in patients with MELD $>20$ in the pre-COVID when compared to those in COVID group ((60/67 (89.6\%) versus $10 / 13(76.9 \%))$. This leads to the conclusion mentioned in the article that only less sicker patients did better during the COVID era when compared to sicker patients during preCOVID. This conclusion is based on very short follow-up post-LT. However, if we look at 1-year survival rates (still COVID era in the study region), based on the MELD cutoff of 20, the rates are comparable (MELD $<20(90 \%)$ vs MELD $>20(84 \%)(P=0.2))$. Therefore, it is safe to say that in parts of the globe where LDLT is the predominant form of transplant (as this study), LDLT can be carried out safely with extra precaution irrespective of the severity of baseline patient characteristics, especially if the population is below 50 years.

\section{References}

1. Bhatti $\mathrm{ABH}$, Nazish M, Khan NY, et al. Living Donor Liver Transplantation During the COVID-19 Pandemic: an Evolving Challenge. J Gastrointest Surg. Jun 15 2021:1-7. https://doi.org/ 10.1007/s11605-021-05057-3

2. Ito T, Naini BV, Markovic D, et al. Ischemia-reperfusion injury and its relationship with early allograft dysfunction in liver transplant patients. Am J Transplant. Feb 2021;21(2):614-625. https:// doi.org/10.1111/ajt.16219

3. Colmenero J, Rodríguez-Perálvarez M, Salcedo M, et al. Epidemiological pattern, incidence, and outcomes of COVID-19 in liver transplant patients. J Hepatol. Jan 2021;74(1):148-155. https:// doi.org/10.1016/j.jhep.2020.07.040

Publisher's Note Springer Nature remains neutral with regard to jurisdictional claims in published maps and institutional affiliations. 